\title{
The importance of special relativistic effects in modelling ultra-fast outflows
}

\author{
A. Luminari ${ }^{1,2}$, F. Tombesi ${ }^{1,3,4,2}$, E. Piconcelli ${ }^{2}$, F. Nicastro ${ }^{2}$, K. Fukumura ${ }^{5}$, D. Kazanas ${ }^{4}$, \\ F. Fiore ${ }^{6}$, and L. Zappacosta ${ }^{2}$ \\ 1 Department of Physics, University of Rome "Tor Vergata", Via della Ricerca Scientifica 1, 00133 Rome, Italy \\ 2 INAF - Osservatorio Astronomico di Roma, Via Frascati 33, 00078 Monteporzio, Italy \\ e-mail: alfredo. luminari@roma2 .infn.it \\ 3 Department of Astronomy, University of Maryland, College Park, MD 20742, USA \\ ${ }^{4}$ NASA/Goddard Space Flight Center, Code 662, Greenbelt, MD 20771, USA \\ 5 Department of Physics and Astronomy, James Madison University, Harrisonburg, VA 22807, USA \\ ${ }^{6}$ INAF - Osservatorio Astronomico di Trieste, via G.B. Tiepolo 11, 34131 Trieste, Italy
}

Received 27 September 2019 / Accepted 25 November 2019

\begin{abstract}
Context. Outflows are observed in a variety of astrophysical sources. Remarkably, ultra-fast $(v \geq 0.1 c)$, highly ionised outflows in the UV and X-ray bands are often seen in active galactic nuclei (AGNs). Depending on their kinetic power and mass outflow rate, $\dot{E}_{\text {out }}$ and $\dot{M}_{\text {out }}$, respectively, these outflows may play a key role in regulating the AGN-host galaxy co-evolution process through cosmic time and metal-feeding the surrounding circum-/inter-galactic medium. It is therefore crucial to provide accurate estimates of the wind properties, including $\dot{M}_{\text {out }}$ and $\dot{E}_{\text {out }}$.

Aims. Here we concentrate on special relativistic effects concerning the interaction of light with matter moving at relativistic speed relatively to the source of radiation. Our aim is to assess the impact of these effects on the observed properties of the outflows and implement a correction for these effects in the existing spectral modelling routines.

Methods. We define a simple procedure to incorporate relativistic effects in radiative transfer codes. Following this procedure, we run a series of simulations to explore the impact of relativistic effects for different outflow velocities and column densities.

Results. The observed optical depth of the wind is usually considered a proxy for its column density $N_{\mathrm{H}}$, independently of the velocity of the outflow. However, our simulations show that the observed optical depth of an outflow with a given $N_{\mathrm{H}}$ decreases rapidly as the velocity of the wind approaches relativistic values. This, in turn, implies that when estimating $N_{\mathrm{H}}$ from the optical depth, it is necessary to include a velocity-dependent correction, already for moderate velocities (e.g. $v_{\text {out }} \gtrsim 0.05 c$ ). This correction linearly propagates to the derived quantities $\dot{M}_{\text {out }}$ and $\dot{E}_{\text {out }}$.

Conclusions. We demonstrate that special relativistic effects must be considered in order to obtain correct estimates of $\dot{M}_{\text {out }}$ and $\dot{E}_{\text {out }}$ for an outflow moving at a mildly relativistic speed relative to the illuminating source of radiation. As an example, we calculate the relativistically corrected values of $\dot{M}_{\text {out }}$ and $\dot{E}_{\text {out }}$ for a sample of $\sim 30$ ultra-fast outflows (UFOs) taken from the literature and find correction factors of $20-120 \%$ within the observed range of outflowing velocities $\left(v_{\text {out }} \approx 0.1-0.3 c\right)$. This brings the ratio between $\dot{M}_{\text {out }}$ and the disc accretion rate close or even above unity for the vast majority of the sources of the sample, highlighting the importance of the reported relativistic corrections to understand the growth of the most massive black holes. The next generation of high-sensitivity X-ray telescopes such as XRISM and Athena will provide a much more complete census of UFOs, especially in the fastest velocity regime where the relativistic corrections are increasingly important.
\end{abstract}

Key words. line: profiles - opacity - radiative transfer - relativistic processes - quasars: absorption lines - accretion, accretion disks

\section{Introduction}

Outflows are ubiquitously observed from a variety of astrophysical sources, and their impact on the surrounding environment depends on their energy. In particular, mildly relativistic and ionised outflows from the innermost regions of active galactic nuclei (AGNs) are often seen in UV and X-ray absorption spectra (e.g. Chartas et al. 2002; Tombesi et al. 2010; Rodríguez Hidalgo et al. 2011; Bruni et al. 2019) and may carry sufficient energy to regulate both the growth of the central supermassive black hole (SMBH) and the evolution of the surrounding host galaxy (Costa et al. 2014; Faucher-Giguère \& Quataert 2012; Tombesi et al. 2015; Zubovas \& King 2012). This critically depends on the kinetic power of these outflows, which in turn depends on their velocity and on their mass flux (Di Matteo et al. 2005; King \& Pounds 2015).

The line-of-sight (LOS) velocity is typically inferred via the blueshift of the absorption features imprinted by the outflowing material onto the continuum emission of the central source, compared to the systemic redshift of the host galaxy. The mass outflow rate $\dot{M}_{\text {out }}$, instead, for a given covering factor and distance of the outflow, is estimated by measuring the optical depth of the absorption features. The observed optical depth is considered a proxy of the outflow column density $N_{\mathrm{H}}$ along the LOS, independently of its outflow velocity $v_{\text {out }}$.

In this work we show that this assumption no longer holds for outflows escaping the central continuum source of radiation with velocities corresponding to a fraction of the speed of light $c$ (e.g. 
$v_{\text {out }} \gtrsim 0.05 c$ ). For these outflows the observed (i.e. apparent) optical depth of the spectral features produced by the absorbing material significantly underestimates the intrinsic $N_{\mathrm{H}}$ and, consequently, the mass transfer rate of the outflows. Therefore, a velocity-dependent correction must be adopted in the estimate of $N_{\mathrm{H}}$ to account for this effect.

This pure special-relativistic effect is universal (i.e. it applies to any fast-moving outflow intercepting the LOS), and affects not only our estimate of the kinetic power of the outflow, but also the ability of the radiative source to effectively accelerate the outflow outwards. For AGN outflows, this may have deep implications on the feedback mechanism and the co-evolution with respect to the host galaxy (Kormendy \& Ho 2013).

The paper is organised as follows. In Sect. 2 we give an overview of the special relativity treatment for a fast-moving gas embedded in a radiation field. In Sect. 3 we show how to incorporate this treatment in modelling outflow spectra. In Sect. 4 we discuss the results and their implications on estimating $\dot{M}_{\text {out }}$ and $\dot{E}_{\text {out }}$, and we summarise in Sect. 5 .

\section{Special relativistic transformation in the outflow reference frame}

According to special relativity, the luminosity $L^{\prime}$ seen by a clump of gas moving at relativistic speed is reduced of a factor $\Psi$, with respect to a static gas, as

$L^{\prime}=L \cdot \Psi$,

where $L$ is the luminosity seen by an observer at rest and $\Psi$ (i.e. the de-boosting factor) is defined as

$\Psi \equiv \psi^{4}=\frac{1}{\gamma^{4}(1-\beta \cos (\theta))^{4}}$,

where $\gamma \equiv \frac{1}{\sqrt{1-\beta^{2}}}, \beta=v_{\text {out }} / c, v_{\text {out }}$ is the gas velocity, and $\theta$ is the angle between the incident luminosity $L$ and the direction of motion of the gas. Figure 1 shows $\Psi$ as a function of $v_{\text {out }}$ for $\theta=180^{\circ}$, corresponding to a radial outward motion of the gas. The deboosting factor is due to the combination of the spacetime dilatation in the gas reference frame $K^{\prime}$, and the relativistic Doppler shift of the received radiation (Rybicki \& Lightman 1986).

Using Eq. (1), the radiative intensity (i.e. the luminosity per solid angle) $\frac{\mathrm{d} L^{\prime}}{\mathrm{d} \Omega^{\prime}}$ received by the outflowing gas in $K^{\prime}$ can be written as a function of the intensity in the rest frame $K$, as

$\frac{\mathrm{d} L^{\prime}}{\mathrm{d} \Omega^{\prime}}=\Psi \frac{\mathrm{d} L}{\mathrm{~d} \Omega}=\psi \mathrm{d} E \cdot \psi^{3} \frac{1}{\mathrm{~d} t \mathrm{~d} \Omega}$

where $\mathrm{d} E, \mathrm{~d} t$, and $\mathrm{d} \Omega$ corresponds to the energy, time, and solid angle intervals in $K$. Specifically, in Eq. (3), $\psi \mathrm{d} E$ is the energy transformation term, which represents the Doppler shift of the wavelengths in $K^{\prime}$. The second term, $\psi^{3} \frac{1}{\mathrm{~d} t \mathrm{~d} \Omega}$, indicates a reduction of the intensity due to the space-time dilatation in $K^{\prime}$.

It should be noted that Eqs. (1) and (3) also describe the emission from gas moving at relativistic velocity, as usually observed in high-velocity systems such as jets in blazars and gamma-ray burts (Urry \& Padovani 1995; Ghisellini et al. 1993). When radiation is emitted along the direction of motion (i.e. $\theta \approx 0^{\circ}$ ), $\Psi$ increases with increasing $v_{\text {out }}$, while $\Psi \leq 1$ when it is emitted perpendicularly or backward $\left(\theta=90^{\circ}\right.$ and $180^{\circ}$, respectively). The overall result is to concentrate the emitted radiation into a narrow cone along the direction of motion, an

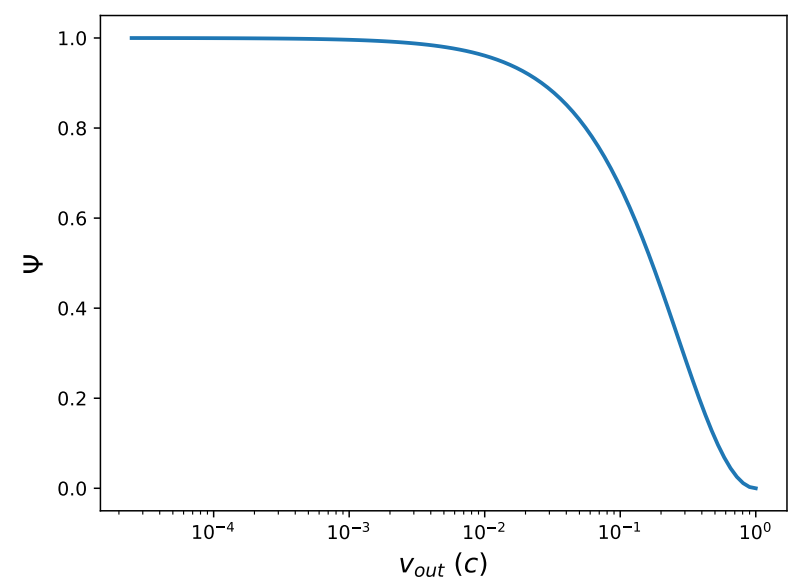

Fig. 1. Deboosting factor $\Psi$ in the gas reference frame $K^{\prime}$ as a function of $v_{\text {out }}$ assuming $\theta=180^{\circ}$. For speeds lower than $0.1 c$, the radiation intercepted by the outflow and by the (rest-frame) observer at infinity are virtually the same. For higher speeds, the fraction of intercepted radiation drops dramatically due to special relativistic effects.

effect known as relativistic beaming (Rybicki \& Lightman 1986; Event Horizon Telescope Collaboration 2019).

Another way of describing the reduction of the luminosity seen by the outflowing gas is the following. In $K^{\prime}$, the luminosity source appears to be moving away with velocity $v_{\text {out }}$ and $\theta=180^{\circ}$ (for a pure radial motion), which results in a deboosting of the received luminosity due to the relativistic beaming, according to Eq. (1).

\section{Modelling outflow absorption spectra including special relativistic effects}

We include these special relativistic corrections in modelling spectral absorption features from the outflowing gas, according to the following procedure (see Appendix A for a detailed description):

- The incident spectrum $S_{\mathrm{I}}(K)$ is transformed from $K$ to $K^{\prime}$, obtaining $S_{\mathrm{I}}\left(K^{\prime}\right)$, according to Eq. (3);

- $S_{\mathrm{I}}\left(K^{\prime}\right)$ is given as input to the radiative transfer code to calculate the transmitted spectrum in the outflowing gas frame $K^{\prime}, S_{\mathrm{T}}\left(K^{\prime}\right)$;

- The relativistic-corrected transmitted spectrum in $K$ (i.e. $\left.S_{\text {out }}(K)\right)$ is given by

$$
S_{\text {out }}(K)=S_{\mathrm{I}}(K) \cdot \Delta+S_{\mathrm{T}}\left(K^{\prime}\right) \cdot \psi^{-1},
$$

where $\Delta \equiv 1-\psi^{3}$. The term $S_{\mathrm{T}}\left(K^{\prime}\right) \cdot \psi^{-1}$ indicates the spectrum $S_{\mathrm{T}}\left(K^{\prime}\right)$ in Doppler-shifted frequencies (from $K^{\prime}$ to $K$ ).

We note that in the low-velocity limit $v_{\text {out }} \ll c, \Psi \approx 1, \Delta \approx 0$, and the resulting spectrum is $S_{\text {out }}(K)=S_{\mathrm{T}}\left(K^{\prime}\right) \cdot \psi^{-1}$, as is usually calculated. For the opposite high-velocity regime $v_{\text {out }} \rightarrow c$, $\Psi \approx 0$, and the outflowing gas does not interact with the ionising radiation. In fact, $S_{\mathrm{I}}\left(K^{\prime}\right)$ and $S_{\mathrm{T}}\left(K^{\prime}\right)$ have null intensity (see Eq. (3)), $\Delta \approx 1$, and $S_{\text {out }}(K) \approx S_{\mathrm{I}}(K)$.

We use the radiative transfer code XSTAR, v2.5 (Kallman \& Bautista 2001) to calculate $S_{\text {out }}(K)$, which is the spectrum as seen by a rest frame observer in $K$.

Figure 2 shows the X-ray spectrum in the range $6-16 \mathrm{keV}$ of a power-law continuum source with $\Gamma=2$ and an ionising luminosity $L_{\text {ion }}=5 \times 10^{46} \mathrm{erg} \mathrm{s}^{-1}$ in the $1-1000 \mathrm{Ry}(1 \mathrm{Ry}=13.6 \mathrm{eV})$ energy interval, modified by an absorber with $v_{\text {out }}=0.0,0.3$, and $0.5 c$. In all cases, we assume an absorbing column density of 

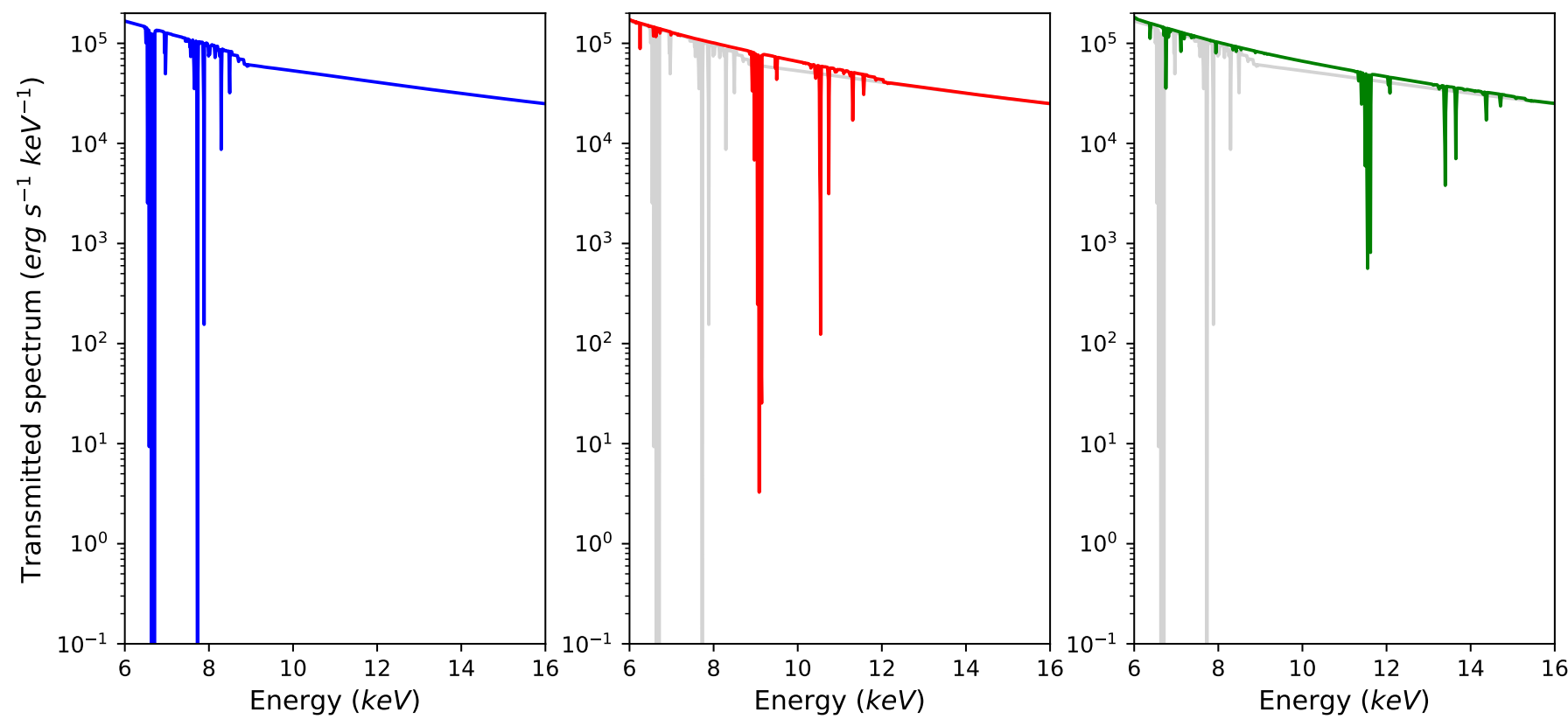

Fig. 2. Absorption spectra for increasing outflow velocity: $v_{\mathrm{out}}=0.00$ (left panel), $=0.30$ (centre panel), $=0.50 \mathrm{c}$ (right panel). For comparison, in the centre and right panels we show the absorption spectrum for $v_{\text {out }}=0.00 c$ (in light grey). See Sect. 3 for details on the spectral parameters used in this simulation.

$N_{\mathrm{H}}=6 \times 10^{23} \mathrm{~cm}^{-2}$ and an ionisation parameter $\log \left(\frac{\xi}{\mathrm{erg} \mathrm{cm} \mathrm{s}^{-1}}\right)=$ 4.5 , which are typical of ultra-fast outflows (UFOs) observed in AGNs (Reeves et al. 2009; Tombesi et al. 2011; Gofford et al. 2013). The middle and right panels of Fig. 2 also report the $v_{\text {out }}=$ 0 case for an easier comparison. It can be seen that the absorption features related to the relativistically outflowing gas are both blueshifted and are significantly weaker than the $v=0$ case. This effect dramatically increases for increasing velocity, as shown in a more quantitative way in Fig. 3, which displays the column density $N_{\mathrm{H}}$ necessary to reproduce outflow absorption features with a fixed optical depth, as a function of $v_{\text {out }}$. The required column density corresponds to $N_{\mathrm{H}}=10^{23} \mathrm{~cm}^{-2}$ for $v_{\text {out }}=0$, and it increases by an order of magnitude for $v_{\text {out }}=0.8 c$. It is interesting to note that this effect may introduce an observational bias in current X-ray data, which are typically restricted to $E<10 \mathrm{keV}$, making outflows at higher velocity more difficult to detect due to the weakening of their spectral features at $E<10 \mathrm{keV}$.

We also note that Schurch \& Done (2007) and Saez \& Chartas (2011) presented AGN outflow models including special relativistic effects to provide an estimate of both $N_{\mathrm{H}}$ and $\xi$. However, the two studies seem not to account properly for the reduction of the optical depth in the calculation of $S_{\text {out }}(K)$. In Eq. (4) the relativistic-corrected optical depth of the wind is preserved by transforming the transmitted spectrum back to the source rest frame $K$, while this aspect has not been considered in these studies.

\section{Discussion}

Mass and kinetic energy transfer rates of the outflow $\left(\dot{M}_{\text {out }}\right.$ and $\dot{E}_{\text {out }}$, respectively), linearly depends on $N_{\mathrm{H}}$. Specifically, $\dot{M}_{\text {out }}$ can be calculated as (Crenshaw \& Kraemer 2012)

$\dot{M}_{\text {out }}=4 \pi r N_{\mathrm{H}} \mu m_{\mathrm{p}} C_{\mathrm{f}} v_{\text {out }}$,

where $r, \mu, m_{\mathrm{p}}$, and $C_{\mathrm{f}}$ are the distance from the source, the mean atomic weight $(\approx 1.4$ for solar abundances), the proton mass,

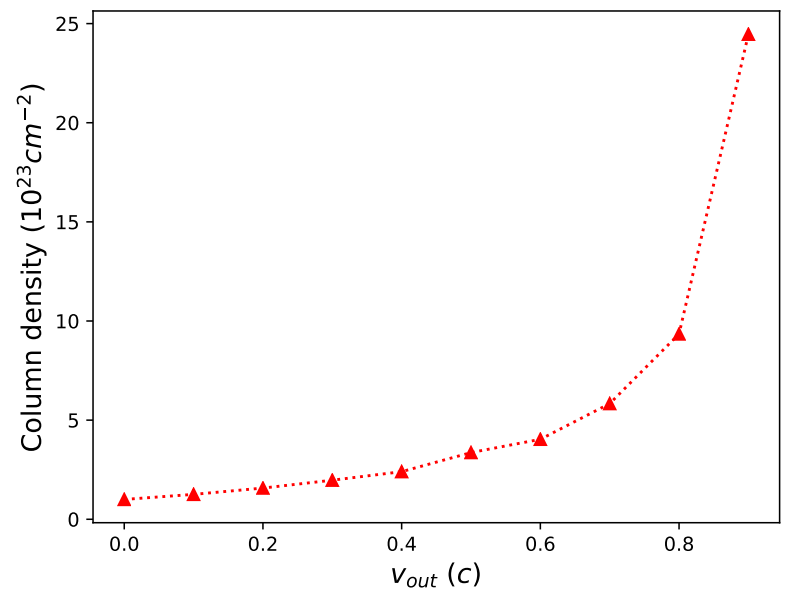

Fig. 3. Absorbing gas $N_{\mathrm{H}}$ required to reach a given value of the optical depth as a function of $v_{\text {out }}$. Spectral parameters are as in Fig. 2.

and the covering factor of the outflow, respectively, and $\dot{E}_{\text {out }}$ is defined as $\dot{E}_{\text {out }}=\frac{1}{2} \dot{M}_{\text {out }} v_{\text {out }}^{2}$. Correct estimates of $\dot{M}_{\text {out }}$ and $\dot{E}_{\text {out }}$ are of fundamental importance to testing theoretical models of two-phase expansion of AGN outflows towards galaxy scales, in which kiloparsec-scale galactic outflows are the result of the shock of ultra-fast, accretion disc-scale outflows onto the ISM (Faucher-Giguère \& Quataert 2012; Zubovas \& King 2012; Menci et al. 2019).

We find that neglecting special relativistic effects will result in an underestimate of $N_{\mathrm{H}}$ and, in turn, of $\dot{M}_{\text {out }}$ and $\dot{E}_{\text {out }}$. As an example, we correct for these effects in the reported values of $\dot{M}_{\text {out }}, \dot{E}_{\text {out }}$ for the UFOs observed in AGNs from Gofford et al. (2015) and Fiore et al. (2017) (see Fig. 3). Specifically, for the UFOs in Gofford et al. (2015) we use the average values between the reported $\dot{M}_{\text {out }}, \dot{E}_{\text {out }}$ calculated using $r_{\min }$ and those using $r_{\max }$, where $r_{\min }\left(r_{\max }\right)$ is the minimum (maximum) inferred launching radius. Values of $\dot{M}_{\text {out }}, \dot{E}_{\text {out }}$ reported in Fiore et al. (2017) are calculated in the same way. In Fig. 4 we plot the ratio of the 


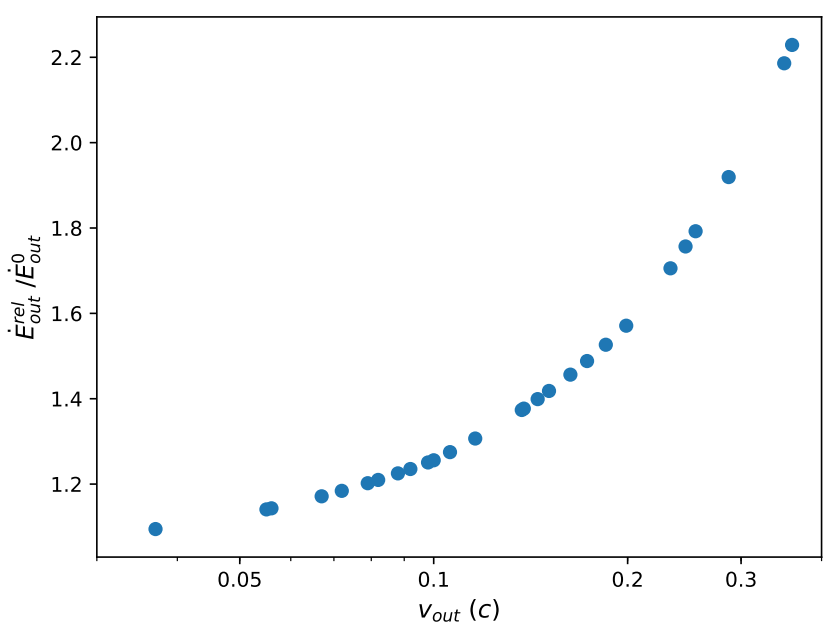

Fig. 4. Ratio of the relativistic-corrected energy transfer rates $\dot{E}_{\text {out }}^{\text {rel }}$ to the original values $\dot{E}_{\text {out }}^{0}$ as a function of $v_{\text {out }}$, for a sample of ultra-fast outflows observed in AGNs (see Sect. 4 for details).

relativistic-corrected energy rates $\dot{E}_{\text {out }}^{\text {rel }}$ to the original values $\dot{E}_{\text {out }}^{0}$ as a function of $v_{\text {out }}$. The value of $\dot{E}_{\text {out }}^{\text {rel }}$ is a factor of $>2$ higher than $\dot{E}_{\text {out }}^{0}$ for the fastest observed outflows $\left(v_{\text {out }} \geq 0.3 c\right)$.

As shown in Fig. 3, we expect even higher ratios for higher velocity outflows. In this respect, the improved sensitivity and resolution of the new generation of X-ray telescopes, such as XRISM and Athena, will be particularly promising and it will allow us to partially overcome the observational bias discussed in Sect. 3. Interestingly, evidence for velocities $\geq 0.4-0.5 c$ have indeed already been reported for some high-luminosity quasars, such as PDS 456 and APM 08279+5255 (see e.g. Reeves et al. 2018; Chartas et al. 2009).

Figure 5 shows the ratio of the relativistic-corrected mass loss rate $\dot{M}_{\text {out }}^{\text {rel }}$ to the mass accretion rate $\dot{M}_{\text {acc }}$, as a function of $\lambda_{\text {Edd }} \equiv L_{\text {bol }} / L_{\text {Edd }}$ (i.e. the ratio of the bolometric and Eddington luminosities). We derive the mass accretion rate as $\dot{M}_{\text {acc }}=\frac{L_{\mathrm{Bol}}}{\eta c^{2}}$, assuming $\eta=0.1$ as in a standard Shakura \& Sunyaev (1973) accretion disc. We note that for almost half of the sources $\frac{\dot{M}_{\text {out }}^{\text {rel }}}{\dot{M}_{\text {acc }}} \geq$ 1, indicating that $\dot{M}_{\text {out }}^{\text {rel }}$ is comparable to (or higher than) the mass accretion rate of the disc. This may indicate a limit for the outflow lifetime, after which the accretion disc is depleted and it can no longer sustain the outflow (see e.g. Belloni et al. 1997). The plot also shows an apparent lack of sources with $\frac{\dot{M}_{\text {out }}^{\text {rel }}}{\dot{M}_{\text {acc }}}>1$ at high $\lambda_{\text {Edd }}$. However, the sample is too small to allow us to draw any conclusions. Future observations of high $\lambda_{\text {Edd }}$ AGNs are needed to shed light on this aspect.

Finally, we compare the outflow momentum rate, defined as $\dot{P}_{\text {out }}=\dot{M}_{\text {out }} v_{\text {out }}$, with the momentum rate of the radiation of the AGN (i.e. $\dot{P}_{\text {rad }}=\frac{L_{\text {bol }}}{c}$ ). We obtain a median $\frac{\dot{P}_{\text {out }}}{\dot{P}_{\text {rad }}}$ value of 0.64 for the original sample, and 0.96 after the relativistic correction. Interestingly, the latter value is consistent with unity, as expected for outflows accelerated through the continuum radiation pressure (the so-called Eddington winds; see e.g. King \& Pounds 2015).

It is worth noting that in Eq. (2) we use the total gas velocity $v_{\text {out }}$. We assume that the velocity $v_{\text {los }}$ along the LOS coincides with $v_{\text {out }}$. As a result, the derived relativistic correction must be regarded as a conservative limit. The correction would increase in the presence of an additional velocity component $v_{\perp}$ perpendicular to the LOS, which implies $v_{\text {out }}=\sqrt{v_{\text {los }}^{2}+v_{\perp}^{2}}$.

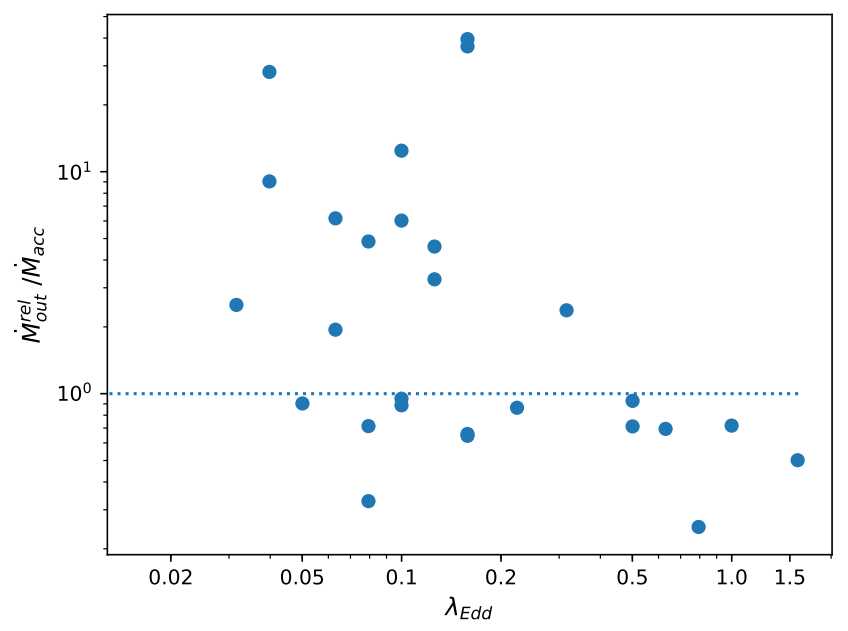

Fig. 5. Ratio of the relativistic-corrected outflow mass rate $\dot{M}_{\text {out }}^{\text {rel }}$ to the inflow mass rate $\dot{M}_{\text {acc }}$, as a function of $\lambda_{\text {Edd }} \equiv L_{\text {bol }} / L_{\text {Edd }}$. The sample is as in Fig. 4. The dotted line corresponds to $\frac{\dot{M}_{\text {out }}^{\text {rel }}}{\dot{M}_{\text {acc }}}=1$.

As an example, we consider the MHD model presented by Fukumura et al. (2010, 2014), where the outflowing gas is launched from the accretion disc at Keplerian velocity. Close to the launching radius, most of the velocity is in the direction of the disc rotation $\phi$, and it is converted to radial velocity at higher distances (i.e. close to the Alfven point) thanks to MHD effects. For a wind launched at $r_{0}=10 r_{\mathrm{G}}{ }^{1}$, the rotational speed has a roughly constant value of $v_{\phi}=0.3 c$ until $r \approx 100 r_{\mathrm{G}}$, while the radial velocity (i.e. the component parallel to the LOS) has an average value of $v_{\mathrm{LOS}} \approx 0.2 c$. In Fig. 1 we show that when $v_{\text {out }}=0, \Psi=1$ and the relativistic effects are absent. On the other hand, when $v_{\text {out }} \rightarrow c, \Psi \approx 0$ and the effects are the highest. Using $v_{\text {LOS }}$ as a proxy for $v_{\text {out }}$ in Eq. (2) yields $\Psi=0.8$, while using the total velocity (i.e. the composition between $v_{\mathrm{r}}$ and $v_{\phi}$ ) gives $\Psi=0.6$, a factor of 0.25 lower.

It is worth noting, as Abramowicz et al. (1991) already pointed out, that the observed optical depth of the gas depends on the velocity of the outflow relative to the source of radiation (see their Eqs. (2.1) and (2.2)). Specifically, they concentrated on an outflowing wind that is optically thick with respect to Thompson scattering, and calculated the integrated luminosity of its photosphere. Moreover, Sumitomo et al. (2007) and Fukue \& Sumitomo (2009) considered the impact of special relativistic effects on the emitted radiation from a fast spherical wind in stars and accreting sources, such as quasars and ULXs. These works further underline the importance of relativistic effects for radiation-matter interaction at high speeds, along with the photo-electric and resonant absorption we investigate in this work.

\section{Conclusions}

In this work we have shown that special relativistic effects are of fundamental importance for a correct modelling of the outflow spectral features, even for mildly relativistic velocities $\left(v_{\text {out }} \gtrsim\right.$ $0.05 c$, see Fig. 1). We have also provided a simple procedure that can be implemented in any radiative transfer code to take into account these effects.

1 The gravitational radius $r_{\mathrm{G}}$ is defined as $r_{\mathrm{G}}=G M / c^{2}$, where $G$ is the gravitational constant and $M$ is the black hole mass. 
We observe a significant reduction of the optical depth of the outflowing gas for fixed $N_{\mathrm{H}}$ and increasing $v_{\text {out }}$ (Figs. 2 and 3). This indicates that it is necessary to include a velocitydependent correction when estimating $N_{\mathrm{H}}$ of the outflow from the optical depth derived by spectral fitting. This correction is already significant (a factor of $\approx 0.5$ ) for an outflow velocity of $v_{\text {out }}=0.1 c$ and reaches values of a factor of $\gtrsim 10$ for $v_{\text {out }} \geq 0.8 c$ (see Fig. 3).

The derived mass and kinetic energy transfer rates linearly depend on $N_{\mathrm{H}}$ (see Eq. (5)), and hence must be corrected accordingly. For AGN outflows, this correction can significantly increase both $\dot{M}_{\text {out }}$ and $\dot{E}_{\text {out }}$ and, in turn, the impact of the outflow on the surrounding environment and on the feedback mechanism. We plot in Figs. 4 and 5 the relativistic-corrected $\dot{E}_{\text {out }}, \dot{M}_{\text {out }}$ for a sample of ultra-fast outflows (UFOs) in AGNs reported in the literature. These pictures further underline the importance of relativistic corrections for a correct assessment of the outflow properties. Furthermore, these corrections are increasingly important in view of the next generation of highsensitivity X-ray telescopes, which will increase the accuracy of the detection of mildly relativistic outflows, as discussed in Sect. 4.

The effects discussed in Sect. 2 have further implications on the radiative driving exerted on the outflowing gas, which will be discussed in a separate work (Luminari et al., in prep.). Moreover, we also plan to present a new version of the X-ray spectral modelling code WINE (Luminari et al. 2018), which includes a relativistic-corrected radiative transfer treatment according to the procedure of Sect. 3.

Acknowledgements. We thank Stefano Ascenzi for useful discussions and Tim Kallman for having provided custom XSTAR packages. AL, EP, FT, LZ acknowledge financial support from the Italian Space Agency (ASI) under the contract ASI-INAF n.2017-14-H.0. FT acknowledges support by the Programma per Giovani Ricercatori - anno 2014 "Rita Levi Montalcini". FF acknowledges support from INAF under the contract PRIN-INAF-2016 FORECAST, and ASI/INAF contract $\mathrm{I} / 037 / 12 / 0$.

\section{References}

Abramowicz, M. A., Novikov, I. D., \& Paczynski, B. 1991, ApJ, 369, 175 Belloni, T., Méndez, M., King, A. R., et al. 1997, ApJ, 488, L109 Bruni, G., Piconcelli, E., Misawa, T., et al. 2019, A\&A, 630, A111 Chartas, G., Brandt, W. N., Gallagher, S. C., et al. 2002, ApJ, 579, 169 Chartas, G., Saez, C., Brandt, W. N., et al. 2009, ApJ, 706, 644

Costa, T., Sijacki, D., \& Haehnelt, M. G. 2014, MNRAS, 444, 2355 Crenshaw, D. M., \& Kraemer, S. B. 2012, ApJ, 753, 75

Di Matteo, T., Springel, V., \& Hernquist, L. 2005, Nature, 433, 604

Event Horizon Telescope Collaboration (Akiyama, K., et al.) 2019, ApJ, 875, L1

Faucher-Giguère, C.-A., \& Quataert, E. 2012, MNRAS, 425, 605

Feruglio, C., Fiore, F., Carniani, S., et al. 2015, A\&A, 583, A99

Fiore, F., Feruglio, C., Shankar, F., et al. 2017, A\&A, 601, A143

Fukue, J., \& Sumitomo, N. 2009, PASJ, 61, 615

Fukumura, K., Kazanas, D., Contopoulos, I., et al. 2010, ApJ, 715, 636

Fukumura, K., Tombesi, F., Kazanas, D., et al. 2014, ApJ, 780, 120

Ghisellini, G., Padovani, P., Celotti, A., et al. 1993, ApJ, 407, 65

Gofford, J., Reeves, J. N., Tombesi, F., et al. 2013, MNRAS, 430, 60

Gofford, J., Reeves, J. N., McLaughlin, D. E., et al. 2015, MNRAS, 451, 4169

Kallman, T., \& Bautista, M. 2001, ApJS, 133, 221

King, A., \& Pounds, K. 2015, ARA\&A, 53, 115

Kormendy, J., \& Ho, L. C. 2013, ARA\&A, 51, 511

Luminari, A., Piconcelli, E., Tombesi, F., et al. 2018, A\&A, 619, A149

Menci, N., Fiore, F., Feruglio, C., et al. 2019, ApJ, 877, 74

Rodríguez Hidalgo, P., Hamann, F., \& Hall, P. 2011, MNRAS, 411, 247

Reeves, J. N., O’Brien, P. T., Braito, V., et al. 2009, ApJ, 701, 493

Reeves, J. N., Braito, V., Nardini, E., et al. 2018, ApJ, 854, L8

Rybicki, G. B., \& Lightman, A. P. 1986, Radiative Processes in Astrophysics, Chapter 4

Saez, C., \& Chartas, G. 2011, ApJ, 737, 91

Schurch, N. J., \& Done, C. 2007, MNRAS, 381, 1413
Shakura, N. I., \& Sunyaev, R. A. 1973, A\&A, 500, 33

Sumitomo, N., Nishiyama, S., Akizuki, C., et al. 2007, PASJ, 59, 1043

Tombesi, F., Cappi, M., Reeves, J. N., et al. 2010, A\&A, 521, A57

Tombesi, F., Cappi, M., Reeves, J. N., et al. 2011, ApJ, 742, 44

Tombesi, F., Meléndez, M., Veilleux, S., et al. 2015, Nature, 519, 436

Urry, C. M., \& Padovani, P. 1995, PASP, 107, 803

Zubovas, K., \& King, A. 2012, ApJ, 745, L34

\section{Appendix A: Algorithm for special relativity corrections}

In this appendix we provide a detailed description of the procedure for special relativity corrections outlined in Sect. 3. Following Eq. (3), the incident spectrum in the outflowing gas reference frame $S_{\mathrm{I}}\left(K^{\prime}\right)$ is obtained from $S_{\mathrm{I}}(K)$ by multiplying the frequencies by a factor $\psi$ and the intensity by a factor $\psi^{3}$.

For a given set of outflow parameters $\left(N_{\mathrm{H}}, \log \left(\xi_{0}\right), n_{0}, v_{\text {out }}\right)$, we run a radiative transfer simulation by using $S_{\mathrm{I}}\left(K^{\prime}\right)$ as incident spectrum. As a result we obtain the transmitted spectrum $S_{\mathrm{T}}\left(K^{\prime}\right)$ displaying the absorption features due to the outflowing gas $^{2}$.

We then calculate the difference spectrum as follows:

$S_{\mathrm{T}-\mathrm{I}}\left(K^{\prime}\right)=S_{\mathrm{T}}\left(K^{\prime}\right)-S_{\mathrm{I}}\left(K^{\prime}\right)$

Accordingly, $S_{\mathrm{T}-\mathrm{I}}\left(K^{\prime}\right)$ represents the absorption features produced by the outflowing gas, with the relativistic-corrected optical depth. As the next step we calculate the relativistic-corrected, rest-frame absorbed spectrum as

$S_{\text {out }}(K)=S_{\mathrm{I}}(K)+S_{\mathrm{T}-\mathrm{I}}\left(K^{\prime}\right) \cdot \psi^{-1}$,

where $S_{\mathrm{T}-\mathrm{I}}\left(K^{\prime}\right) \cdot \psi^{-1}$ represents the difference spectrum in rest frame $(K)$ frequencies, which is obtained by dividing the frequencies by a factor $\psi$. Using Eq. (A.1), we can thus rewrite Eq. (A.2) as

$S_{\text {out }}(K)=S_{\mathrm{I}}(K) \cdot \Delta+S_{\mathrm{T}}\left(K^{\prime}\right) \cdot \psi^{-1}$,

where $\Delta \equiv 1-\psi^{3}$ and $S_{\mathrm{I}}(K) \cdot \Delta$ indicates a scaling of the intensity of the spectrum $S_{\mathrm{I}}(K)$ of a factor $\Delta$.

In our calculations we assume that the outflow has a net velocity $v_{\text {out }}$ and direction $\theta$. From a physical point of view, $v_{\text {out }}$ and $\theta$ correspond to the average velocity and direction of the outflow, respectively. Therefore, if a turbulent velocity component is present, the above discussion is still valid, provided that $v_{\text {turb }} \ll v_{\text {out }}$. Furthermore, if the outflowing velocity is a function of the spatial coordinates (i.e. $v_{\text {out }}=v(\vec{r})$ ) the above procedure can be implemented by dividing the outflow into small slabs, and assuming $v_{\text {out }}$ to be constant in each of them. Finally, the treatment of more complicated scenarios for $v(\vec{r})$ requiring a first-principle approach are beyond the scope of the present paper.

\footnotetext{
2 Moreover, in some of the most popular codes, such as XSTAR, the emissivity of all the atomic lines listed in the atomic database is saved in a separated file. Emissivities can be used to build relativistic-corrected outflow emission spectra, as we will illustrate in detail in a forthcoming paper.
} 